\title{
Commensurate transverse helical ordering in the room-temperature magnetoelectric $\mathrm{Co}_{2} \mathrm{Z}$ hexaferrite
}

\author{
Hun Chang ${ }^{\mathrm{a}}$, Hak Bong Lee ${ }^{\mathrm{a}}$, Jae-Ho Chung, ${ }^{\mathrm{a}, *}$, Sae Hwan Chun ${ }^{\mathrm{b}}$, Kwang Woo \\ Shin ${ }^{\mathrm{b}}$, Byung-Gu Jeon ${ }^{\mathrm{b}}$, Kee Hoon Kim ${ }^{\mathrm{b}}$, Karel Prokeš ${ }^{\mathrm{c}}$, Slavomir Mat'ašc \\ ${ }^{a}$ Department of Physics, Korea University, Seoul 02841, South Korea \\ ${ }^{b}$ CeNSCMR, Dept. of Physics and Astronomy, Seoul National University, Seoul 08826, \\ South Korea \\ ${ }^{c}$ Helmholtz-Zentrum für Materialien und Energie, D-14109 Berlin, Germany
}

\begin{abstract}
Using single crystal neutron diffraction, we confirmed the existence of the commensurate transverse helical ordering in $\mathrm{Ba}_{0.52} \mathrm{Sr}_{2.48} \mathrm{Co}_{2} \mathrm{Fe}_{24} \mathrm{O}_{41}$ upon zero-field cooling, in which room-temperature magnetoelectric coupling has been previously observed. Its magnetic structure remained commensurate to the lattice under the external field perpendicular to the $c$ axis. We also found the commensurate ordering to be directly responsible for the electric polarization, which is the common feature of many types of hexaferrites independently of their zerofield orderings.

Keywords: multiferroics, hexaferrites, neutron diffraction, magnetocrystalline anisotropy
\end{abstract}

\section{Introduction}

Hexagonal ferrites have long been utilized for industrial applications by virtue of their high Curie temperatures and flexibility encompassing soft to hard ferrimagnetic behaviors. [1] Their high magnetic ordering temperatures 5 are ascribed to strong superexchanges between large spin moments of $\mathrm{Fe}^{3+}$ ions $\left(3 d^{5}, S=5 / 2\right)$ built upon the Hund's coupling. In contrast, the hard-

\footnotetext{
* Corresponding author

Email address: jaehc@korea.ac.kr (Jae-Ho Chung)
}

Preprint submitted to Physica B

December 2, 2021 
ness of a magnet is determined by magnetocrystalline anisotropy fields arising due to unquenched spin-orbit coupling. The layered hexagonal lattices provide highly anisotropic structural symmetry contributing to significant magnetic anisotropy fields. Combinations of these two features in hexaferrites have recently led to the remarkable property observable at room temperature, namely magnetoelectricity. 2] The magnetoelectric couplings, in which electric polarizations appear via magnetic origin, have usually been observed at cryogenic temperatures since magnetic energies are typically weaker than lattice energies. 3, 4, 5, 6, 7. It was only in the hexaferrites where the finite magnetoelectric polarizations have been achieved at room temperature, which also allow both magnetic-field control of electric polarization $(\Delta P-H)$ and electric-field control of magnetic polarization $(M-E) \cdot[8,9,10]$

There are several structural variants of the hexaferrites, in which $\mathrm{Fe}^{3+}$ ions occupy crystallographic sites with either four or six oxygen coordinations providing tetrahedral or octahedral crystal fields, respectively. [1] The initial observation of the magnetoelectric polarization was realized in the Y-type $\mathrm{Ba}_{0.5} \mathrm{Sr}_{1.5} \mathrm{Zn}_{2} \mathrm{Fe}_{12} \mathrm{O}_{22}$ $\left(\mathrm{Zn}_{2} \mathrm{Y}\right)$ well below room temperature when an external magnetic field was applied perpendicularly to the $c$ axis. 11] Its zero-field magnetic structure is planar helical with incommensurate pitches to the lattice, [12] for which the inverse Dzyalishinskii-Moriya mechanism should not generate finite electric polarizations. [13, 14. Since the planar structure suggests significant easy-plane anisotropy field within $a b$ (or $c$ ) planes, fairly strong magnetic field $(>0.2 \mathrm{~T})$ perpendicular to the $c$ axis was required to induce the electric polarization. [11] The magnitude of the onset field could be reduced by more than an order of magnitude upon replacing a few percent of $\mathrm{Fe}^{3+}$ ions with $\mathrm{Mg}^{2+}, \mathrm{Co}^{2+}$ or $\mathrm{Al}^{3+}$ ions. 15, 16, 17, 18, The resultant reduction of the easy-plane anisotropy simultaneously caused the magnetic ordering to become heliconical with the net moment along the $c$ axis. [16, 19] Finite magnetoelectric polarizations appear when the helicones 35 tilt off the $c$ axis under the external field. [15, 20] Commonly in different types of hexaferrites, these transverse heliconical orderings are usually found to be commensurate to the structural lattices. [16, 20, 19, 21, 22] 
Recently, the room-temperature magnetoelectric coupling was realized in a different type of hexaferrites, namely the Z-type $(\mathrm{Ba}, \mathrm{Sr})_{3} \mathrm{Co}_{2} \mathrm{Fe}_{24} \mathrm{O}_{41}\left(\mathrm{Co}_{2} \mathrm{Z}\right)$. 8,

40 9] Its crystal structure also consists mainly of stacked layers of tetrahedral and octahedral $\mathrm{Fe}^{3+}$ sites shared by $\mathrm{Co}^{2+}\left(3 d^{7}\right)$ ions. The high-temperature magnetoelectric coupling is ascribed to magnetic $\mathrm{Co}^{2+}\left(3 d^{7}\right)$ ions contributing to enhancing the electronic exchange strength. As depicted in Fig. 1(a), its structural layers may be grouped into a few blocks, in each of which spin orientations

45 are expected to be internally collinear similarly as in the Y-type. 18 Whereas the smaller $\mathrm{S}$ blocks are identical, the larger L block of the Z-type is twice thicker than the counterpart in the Y-type structure. The spiral pitch is driven by the exchange competitions near the L-S interfaces that commonly exist in both types. 23. Contrary to the Y-type, however, the previous study based on neutron powder diffraction suggested that no incommensurate heliconical ordering existed for the $\mathrm{Co}_{2} \mathrm{Z}$ in zero field. 24] In this work, we investigated the heliconical magnetic ordering in a single crystal $\mathrm{Ba}_{0.52} \mathrm{Sr}_{2.48} \mathrm{Co}_{2} \mathrm{Fe}_{24} \mathrm{O}_{41}$, particularly focusing on its existence upon zero-field cooling and the evolution in the transverse magnetic field up to $3 \mathrm{~T}$. We find that the magnetic ordering remained to be commensurate throughout the field range investigated.

\section{Experimental}

Single crystalline samples were grown using the flux method that is described in Ref. [9]. The largest obtained piece $(\sim 48 \mathrm{mg})$ was used for the neutron diffraction measurements while smaller crystals with higher quality were used for magnetoelectric measurements. Commonly in all measurements, crystals were cooled in zero field down to the base temperature and magnetic field was then applied perpendicular to the $c$ axis (|| [120]). Electric polarization (\| [100]) was obtained by integrating magnetoelectric current measured following poling simultaneously with $+\mu_{0} H_{p}=2 \mathrm{~T}$ and $+E=230 \mathrm{kV} / \mathrm{m}$.

Single crystal diffraction measurements were performed at the HelmholtzZentrum Berlin für Materialien und Energie using the E4 diffractometer $(\lambda=$ 
(a)

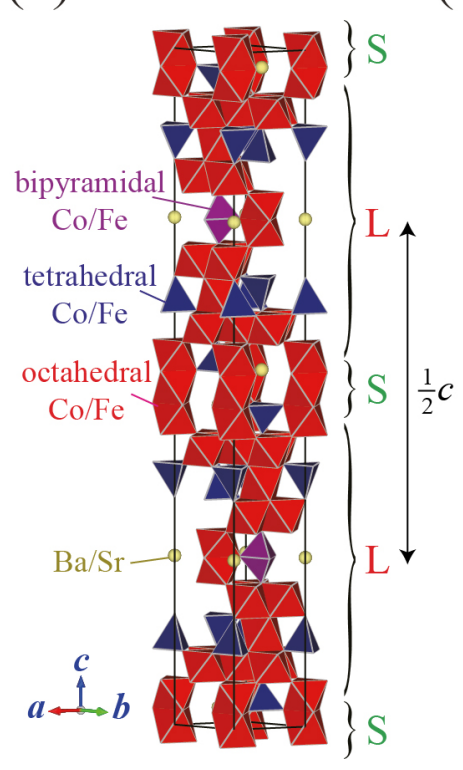

(b)

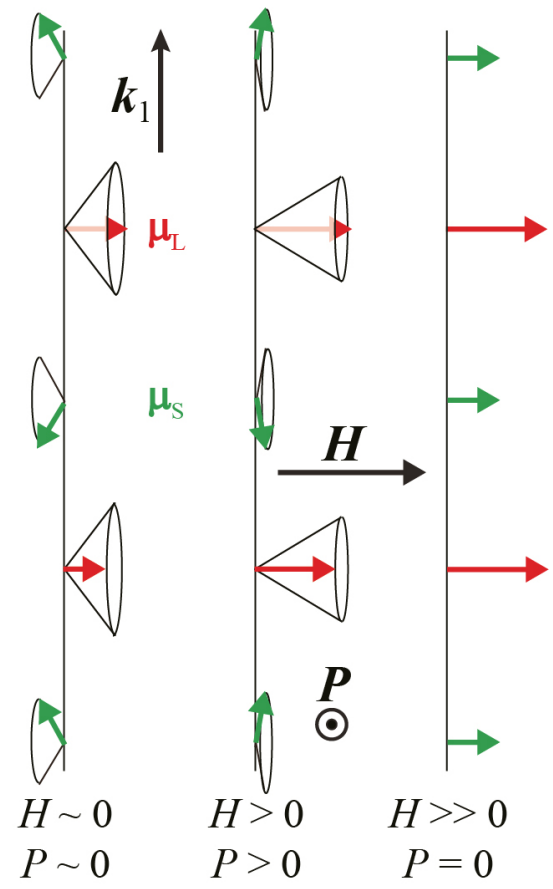

Figure 1: (a) The crystal structure of the $\mathrm{Co}_{2} \mathrm{Z}$ hexaferrites. The black solid lines enclose one structural unit cell. The L and S are the structural blocks according to Ref. 9], in each of which magnetic moments are assumed to be internally collinear. (b) Schematic illustrations of the magnetic ordering in low, intermediate, and high magnetic fields, respectively. Only the net moments of the $\mathrm{L}$ and $\mathrm{S}$ blocks, respectively, are shown. 
$2.43 \AA$ ). The crystal was oriented with the reciprocal $\mathbf{a}^{*}$ and $\mathbf{c}^{*}$ vectors in the horizontal scattering plane before inserting in the superconducting split-pair cryomagnet. Diffraction intensities were collected on the position-sensitive detectors by performing scans in the two-axis mode. The single crystal piece used for neutron diffraction measurements contained three crystallographic domains as well as an Y-type inclusion. While the Bragg reflections from different domains were well separated from each other along $\left[\begin{array}{lll}0 & 0 & l\end{array}\right]$ direction, those along $\left[\begin{array}{lll}1 & 0 & l\end{array}\right]$ reflections were significantly overlapped. For this reason, we used the two-dimensional Gaussian fitting of the collected data in order to separate their individual intensity contributions.

\section{Results and discussions}

We searched for the possible presence of incommensurate heliconical magnetic ordering upon zero-field cooling. Since the neutron scattering will have non-zero cross sections only for magnetic moments perpendicular to the momentum transfer, the magnetic intensities due to incommensurate helical ordering within the $a b$ planes will appear along $\mathbf{Q}=l \mathbf{c}^{*}$ with non-integer values of $l$. Fig. 2(a) shows the single crystal neutron diffraction intensities observed along the $\left[\begin{array}{lll}0 & 0 & l\end{array}\right]$ direction in the reciprocal space during zero-field cooling. From 300

${ }_{85} \mathrm{~K}$ down to $10 \mathrm{~K}$, we found that all observed peaks appeared only at integer values of $l$. No evidence was found for the existence of incommensurate peaks, which is a distinct behavior with respect to the magnetoelectric Y- or M-type hexaferrites. At the same time, overall intensities did not show any noticeable changes within the temperature range investigated. The space group symmetry $\left(P 6_{3} / m m c\right)$ of the Z-type crystal lattice dictates that the structural peaks are forbidden at $00 l$ reflections with $l=2 n+1$ ( $n=$ integers). The intensities observed at these wave vectors thus should be ascribed entirely to the magnetic ordering of $\mathrm{Fe}^{3+}$ spins oriented perpendicular to the $c$ axis. The propagation vector of the associated ordering is $\mathbf{k}_{0}=(0,0,1)$, for which spin orientations are reversed between two planes separated by $\frac{1}{2} c$. This separation corresponds, for 
instance, to the spacing between the centers of two adjacent L (or S) blocks in Fig. 1(a).

We note that the Bragg peaks observed at $l=2 n$ may also include magnetic intensities as well as structural. The propagation vector of the associated magnetic ordering is $\mathbf{k}_{0}=(0,0,0)$, for which spins are oriented parallel between the above-mentioned two planes. Since the magnetic intensities could not be explicitly separated from the structural, instead we observed their intensity changes under the external magnetic field and compared them with the dc magnetization and electric polarization. Fig. 2(b) shows the neutron diffraction intensities at $10 \mathrm{~K}$ under the external magnetic field that was successively ramped up to 3 $\mathrm{T}$ and back down to zero. It is clear that intensities observed at even $l$ values increase under the external field indicating that the $\mathbf{k}_{0}$ component develops accordingly. In the meantime, the intensities at odd $l$ values decrease and finally become invisible at $3 \mathrm{~T}$. No intensity was noticed at incommensurate wave vectors under the external field as well. When the external field was ramped back down to zero, the diffraction intensities were recovered for both sets of Bragg peaks. We thus conclude that the magnetic ordering remained to be commensurate to the structure in all temperatures and fields investigated.

In order to have further insights into the magnetic ordering, in Fig. 3 we 115 plotted the integrated intensities of Bragg peaks under the external field. Also plotted are same figure are the dc magnetization $\left(M_{a b}\right)$ and electric polarization $(\Delta P)$, which were measured along mutually perpendicular directions within the $a b$ planes. Both $M_{a b}$ and $\Delta P$ remained very weak at low fields, and simultaneously exhibited sharp increases under the external field. The neutron diffraction intensities, however, remained almost unchanged in the low field range below 0.05 T. (See Figs. 3(c) and 3(d), respectively.) Since it suggests little changes in the underlying magnetic structure, the observed field dependences in this range are primarily ascribed to in-plane rotations of the magnetic domains. The neutron diffraction intensities finally exhibited noticeable changes when ${ }_{125}$ the magnetic field exceeded $0.05 \mathrm{~T}$, where a kink is observed also in $M_{a b}$. The observed changes in this field rage indicate that the in-plane $\mathbf{k}_{0}$ component of 
the magnetic structure increased at the expense of the $\mathbf{k}_{1}$. Simultaneously, the electric polarization approached its maximum value suggesting that the helical component of the magnetic structure became maximized. As the magnetic field was further increase beyond $2 \mathrm{~T}$, both the intensities of $\left(\begin{array}{lll}0 & 0 & 2 n\end{array}\right)$ reflections and the magnetization finally became saturated whereas those of $\left(\begin{array}{l}0 \\ 0\end{array} 2 n+1\right)$ reflections disappeared. We observe that the electric polarization also became zero in this field range indicating that the heliconical magnetic ordering finally collapsed and became collinear [see Fig. 11(b)]. We find that $\Delta P\left(\mu_{\mathrm{B}} H\right)$ exhibited a dome-like maximum near $0.2 \mathrm{~T}$, which is distinct from the plateau-like maxima observed in Al-doped $\mathrm{Zn}_{2}$ Y-type. [17] It suggests that the helical component of the $\mathrm{Co}_{2} \mathrm{Z}$ experiences relatively weaker pinning force during the field-induce changes near the maximum.

Since the commensurate transverse heliconical ordering must involve magnetic moments along the $c$ axis in addition to those within the $a b$ planes, we expect the $\mathbf{k}_{1}$ component to appear also at Bragg reflections with non-zero $h$ indexes. We thus also observe field-dependent changes in the integrated intensity of $\left(\begin{array}{lll}1 & 0 & 0\end{array}\right)$ Bragg reflection, which is plotted in Fig. 3(e). Contrary to the case of $\left(\begin{array}{ll}0 & 0 l\end{array}\right)$, this reflection includes contributions both from the $\mathbf{k}_{0}$ and $\mathbf{k}_{1}$ orderings that cannot be directly separated. Nevertheless, its intensity exhibited a field-induced decrease between $0.05 \mathrm{~T}$ and $2.0 \mathrm{~T}$, which provides us with an important clue. Since the $\mathbf{k}_{0}$ component must enhance in this range, the apparent decrease in ( $\left(\begin{array}{lll}1 & 0 & 0\end{array}\right)$ intensity should account for the $\mathbf{k}_{1}$ component parallel to the $c$ axis. By combining with the zero-field observation, we thus conclude that the transverse conical ordering exists in zero field. The associated magnetic structure is schematically depicted in Fig. 1(b) using the net magnetic moments of $\mathrm{L}$ and $\mathrm{S}$ spin blocks, or $\mu_{\mathrm{L}}$ and $\mu_{\mathrm{S}}$, respectively. While the $\mathbf{k}_{0}$ ordering is ascribed to the cone axes perpendicular to the $c$ axis, the $\mathbf{k}_{1}$ accounts for the helical component with $90^{\circ}$-pitches between neighboring blocks. We suppose 155 that $\mu_{\mathrm{S}}$ is more likely to be along the $c$ axis because the $\mathrm{S}$ blocks contain more tetrahedral sites. 19] The intensity of the (1 000$)$ reflection eventually was recovered at higher field when the magnetization was saturated, which is consistent 
with the $\mathbf{k}_{0}$ being maximized. A similar saturation was also observed in the (1 0 l 1) reflection [see Fig. 3(f).] When the magnetic field was subsequently ramped down to zero, neutron diffraction intensities were almost fully recovered for all reflections as shown in Fig. 3. Although the dc magnetization showed a weak hysteresis, the small remanent magnetization $\left(0.34 \mu_{\mathrm{B}} / \mathrm{f} . \mathrm{u}.\right)$ suggests a weak in-plane coercivity. We do not expect the associated differences to be observable with neutron diffraction.

As mentioned previously, the commensurate transverse cones are commonly observed among various types of magnetoelectric hexaferrites. They appear in all magnetoelectric phases of the $\mathrm{Y}-, \mathrm{M}-$, and $\mathrm{U}$-types as well as $\mathrm{Co}_{2} \mathrm{Z}$ regardless of initial magnetic orderings upon zero-field cooling. [22, 18, 16, 25, 26, 27] Since the structural periods along the $c$ axes are rather long in many hexaferrites, the antiferromagnetic exchanges may not stabilize such commensurate heliconical orderings. For instance, $180^{\circ}$-pitches are expected between two $a b$ planes separated by $14.5 \AA$ for the Y-type, or by $26.1 \AA$ for the Z-type structures. It has thus been suggested that the two distinct crystal anisotropy fields of the spin blocks play more important roles in locking the commensurate magnetic ordering. [19] What should then be the reason that the transverse conical ordering appears immediately upon zero-field cooling in the Z-type but not in the Y-type? We figure that it is also related to the larger structural period of the Z-type along the $c$ axis. Note that the angles between $\mu_{L}$ and $\mu_{S}$ are determined by the exchange energies across the L-S interfaces as well as two coexisting magnetic anisotropies. The former will primarily affect the in-plane angles whereas those off the planes are determined by the latter. 23. Since the $\mathrm{L}$ and $\mathrm{S}$ blocks are supposed to possess net easy-plane and easy-axis anisotropy fields, respectively, [19] in the commensurate transverse ordering $\mu_{L}$ should lie within the $a b$ planes while $\mu_{S}$ are tilted off (see Fig. 11). In contrast, both $\mu_{L}$ and $\mu_{S}$ should be off the planes for incommensurate heliconical orderings to appear. 18] All involved spins in this case will have similar tilt angles due to the antiferromagnetic couplings between layers, which is the case for the Y-type upon zero-field cooling. [23, 18] For $\mu_{L}$ to be fixed in the planes while $\mu_{S}$ is not, 
however, the tilt angles of the involved spins should gradually increase across

the L block towards the L-S interface. We suppose that such arrangement is achieved in the Z-type because its L block is twice thicker than in the Y-type. This scenario is also consistent with the commensurate Bragg peaks observed in the U-type, in which the $\mathrm{L}$ block is three times thicker than in the Y-type. 27].

\section{Summary and conclusion}

In summary, we have investigated magnetic ordering of $\mathrm{Ba}_{0.52} \mathrm{Sr}_{2.48} \mathrm{Co}_{2} \mathrm{Fe}_{24} \mathrm{O}_{41}$ in zero and finite magnetic fields applied perpendicular to the $c$ axis of a single crystal sample. Magnetic Bragg peaks were observed only at commensurate wave vectors confirming that the transverse heliconical ordering existed in zerofield cooling. When the magnetic field was applied, the electric polarization reached its maximum value without significant changes in the underlying magnetic structures. The helical ordering finally collapsed at high fields coincidently with the suppression of electric polarization. We argue that the thickness of the L block is an important factor in determining the stability of the commensurate transverse heliconical ordering in magnetoelectric hexaferrites.

\section{Acknowledgement}

This research was supported by National R\&D Program through the National Research Foundation of Korea (NRF) funded by the Ministry of Science, ICT and Future Planning (grant number: NRF-2017K1A3A7A09016303; NRF2016R1D1A1B03934157).

\section{References}

[1] J. Smit, H. P. J. Wijn, Ferrites, Philips Technical Library, Eindhoven (1959) Chapter IX. Ferrites with hexagonal.

[2] S.-W. Cheong, M. Mostovoy, Multiferroics: a magnetic twist for ferroelectricity, Nature Mater. 6 (2007) 13-20. doi:10.1038/nmat1804. 
[3] N. Hur, S. Park, P. A. Sharma, J. S. Ahn, S. Guha, S.-W. Cheong, Electric polarization reversal and memory in a multiferroic material induced by magnetic fields, Nature 429 (2004) 392-395. doi:10.1038/nature02572.

[4] G. Lawes, A. B. Harris, T. Kimura, N. Rogado, R. J. Cava, O. E.-W. A. Aharony, T. Yildrim, C. B. M. Kenzelmann, , A. P. Ramirez, Magnetically driven ferroelectric order in $\mathrm{Ni}_{3} \mathrm{~V}_{2} \mathrm{O}_{8}$, Phys. Rev. Lett. 95 (2005) 087205. doi:10.1103/PhysRevLett.95.087205.

[5] M. Kenzelmann, A. B. Harris, S. Jonas, C. Broholm, J. Schefer, S. Kim, C. L. Zhang, S.-W. Cheong, O. P. Vajk, , J. W. Lynn, Magnetic inversion symmetry breaking and ferroelectricity in $\mathrm{TbMnO}_{3}$, Phys. Rev. Lett. 95 (2005) 087206. doi:10.1103/PhysRevLett.95.087206.

[6] K. Taniguchi, N. Abe, Y. I. T. Takenobu, , T. Arima, Ferroelectric polarization flop in a frustrated magnet $\mathrm{MnWO}_{4}$ induced by a magnetic field, Phys. Rev. Lett. 97 (2006) 097203. doi:10.1103/PhysRevLett.97.097203.

[7] Y. Yamasaki, S. Miyasaka, Y. Kaneko, J.-P. He, T. Arima, , Y. Tokura, Magnetic reversal of the ferroelectric polarization in a multiferroic spinel oxide, Phys. Rev. Lett. 96 (2006) 207204. doi:10.1103/PhysRevLett.96. 207204

[8] Y. Kitagawa, Y. Hiraoka, T. Honda, T. Ishikura, H. Nakamura, T. Kimura, Low-field magnetoelectric effect at room temperature, Nature Materials 9 (2010) 797-802. doi:10.1038/nmat2826.

[9] S. H. Chun, Y. S. Chai, B.-G. Jeon, H. J. Kim, Y. S. Oh, I. Kim, H. Kim, B. J. Jeon, S. Y. Haam, J.-Y. Park, S. H. Lee, J.-H. Chung, J.-H. Park, , K. H. Kim, Electric field control of nonvolatile four-state magnetization at room temperature, Phys. Rev. Lett. 108 (2012) 177201. doi:10.1103/ PhysRevLett.108.177201.

[10] J. Wu, Z. Shi, J. Xu, N. Li, Z. Zheng, H. Geng, Z. Xie, L. Zheng, Synthesis 
and room temperature four-state memory prototype of $\mathrm{sr}_{3} \mathrm{Co}_{2} \mathrm{fe}_{2} 4 \mathrm{o}_{4} 1 \mathrm{mul}-$ tiferroics, Appl. Phys. Lett. 101 (2012) 122903. doi:10.1063/1.4753973.

[11] T. Kimura, G. Lawes, A. P. Ramirez, Electric polarization rotation in a hexaferrite with long-wavelength magnetic structures, Phys. Rev. Lett. 94 (2005) 137201. doi:10.1103/PhysRevLett.94.137201.

[12] N. Momozawa, Neutron diffraction study of helimagnet $\left(\mathrm{Ba}_{1-x} \mathrm{Sr}_{x}\right)_{2} \mathrm{Zn}_{2} \mathrm{Fe}_{12} \mathrm{O}_{22}$, J. Phys. Soc. Jpn. 55 (1986) 4007-4013. doi:10.1143/JPSJ.55.4007.

[13] H. Katsura, N. Nagaosa, , A. V. Balatsky, Spin current and magnetoelectric 口 effect in noncollinear magnets, Phys. Rev. Lett. 95 (2005) 057205. doi: 10.1103/PhysRevLett.95.057205.

[14] A. B. Harris, Landau analysis of the symmetry of the magnetic structure and magnetoelectric interaction in multiferroics, Phys. Rev. B 76 (2007) 054447. doi:10.1103/PhysRevB.76.054447.

[15] S. Ishiwata, Y. Taguchi, H. Murakawa, Y. Onose, Y. Tokura, Lowmagnetic-field control of electric polarization vector in a helimagnet, Science 319 (2008) 1643-1646. doi:10.1126/science.1154507

[16] H. B. Lee, S. H. Chun, K. W. Shin, B.-G. Jeon, Y. S. Chai, K. H. Kim, J. Schefer, H. Chang, S.-N. Yun, T.-Y. Joung, , J.-H. Chung, Heliconical magnetic order and field-induced multiferroicity of the $\mathrm{Co}_{2} \mathrm{Y}$ type hexaferrite $\mathrm{Ba}_{0.3} \mathrm{Sr}_{1.7} \mathrm{Co}_{2} \mathrm{Fe}_{12} \mathrm{O}_{22}$, Phys. Rev. B 86 (2012) 094435. doi:10.1103/PhysRevB.86.094435

[17] S. H. Chun, Y. S. Chai, Y. S. Oh, D. Jaiswal-Nagar, S. Y. Haam, I. Kim, B. Lee, D. H. Nam, K.-T. Ko, J.-H. Park, J.-H. Chung, , K. H. Kim, Realization of giant magnetoelectricity in helimagnets, Phys. Rev. Lett. 104 (2010) 037204. doi:10.1103/PhysRevLett.104.037204.

[18] H. B. Lee, Y.-S. Song, J.-H. Chung, S. H. Chun, Y. S. Chai, K. H. Kim, M. Reehuis, K. Prokeš, S. Mat'aš, Field-induced incommensurate- 
[19] H. Chang, H. B. Lee, Y.-S. Song, J.-H. Chung, S. A. Kim, I. H. Oh, M. Reehuis, J. Schefer, Al doping effect on magnetic phase transitions

[21] N. Momozawa, Y. Yamaguch, Field-induced commensurate intermediate phases in helimagnet $\left(\mathrm{Ba}_{1-x} \mathrm{Sr}_{x}\right)_{2} \mathrm{Zn}_{2} \mathrm{Fe}_{12} \mathrm{O}_{22}(x=\mathrm{O} .748)$, J. Phys. Soc. Jpn. 62 (1993) 1292-1304. doi:10.1143/JPSJ.62.1292.

[22] H. Sagayama, K. Taniguchi, N. Abe, T. H. Arima, Y. Nishikawa, S. I.

[24] M. Soda, T. Ishikura, H. Nakamura, Y. Wakabayashi, T. Kimura, Magnetic ordering in relation to the room-temperature magnetoelectric effect

[25] O. P. Aleshko-Ozhevskii, R. A. Sizov, I. I. Yamzin, V. A. Lubimt- 
sev, Helicoidal antiphase spin ordering in hexagonal ferrites of the $\mathrm{BaSc}_{x} \mathrm{Fe}_{12-x} \mathrm{O}_{19}(\mathrm{M})$ system, Soviet Physics JETP 28 (1969) 425.

[26] Y. Tokunaga, Y. Kaneko, D. Okuyama, S. Ishiwata, T. Arima, S. Wakimoto, K. Kakurai, Y. Taguchi, Y. Tokura, Multiferroic $M$-type hexaferrites with a room-temperature conical state and magnetically controllable spin helicity, Phys. Rev. Lett. 105 (2010) 257201. doi:10.1103/PhysRevLett. 105.257201 .

[27] K. Okumura, T. Ishikura, M. Soda, T. Asaka, H. Nakamura, Y. Wakabayashi, T. Kimura, Magnetism and magnetoelectricity of a U-type hexa-

口. ferrite $\mathrm{Sr}_{4} \mathrm{Co}_{2} \mathrm{Fe}_{36} \mathrm{O}_{60}$, Appl. Phys. Lett. 98 (2011) 212504. doi:10.1063/ 1.3593371 . 


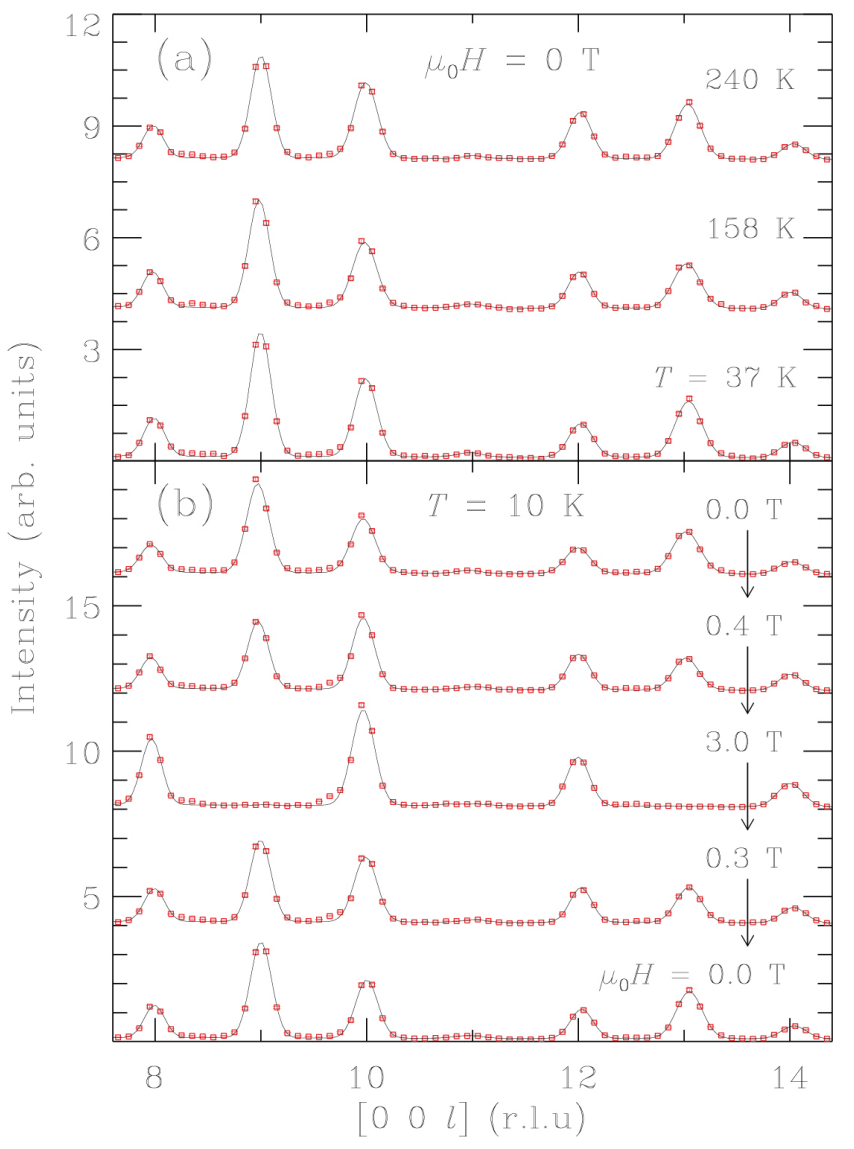

Figure 2: Neutron diffraction intensities observed along the momentum transfer parallel to the $c$ axis (a) at selected temperatures during zero-field cooling or (b) at selected external fields at $T=10 \mathrm{~K}$. In (b), the vertical arrow depicts the sequence of measurements during the field ramping up or down. 


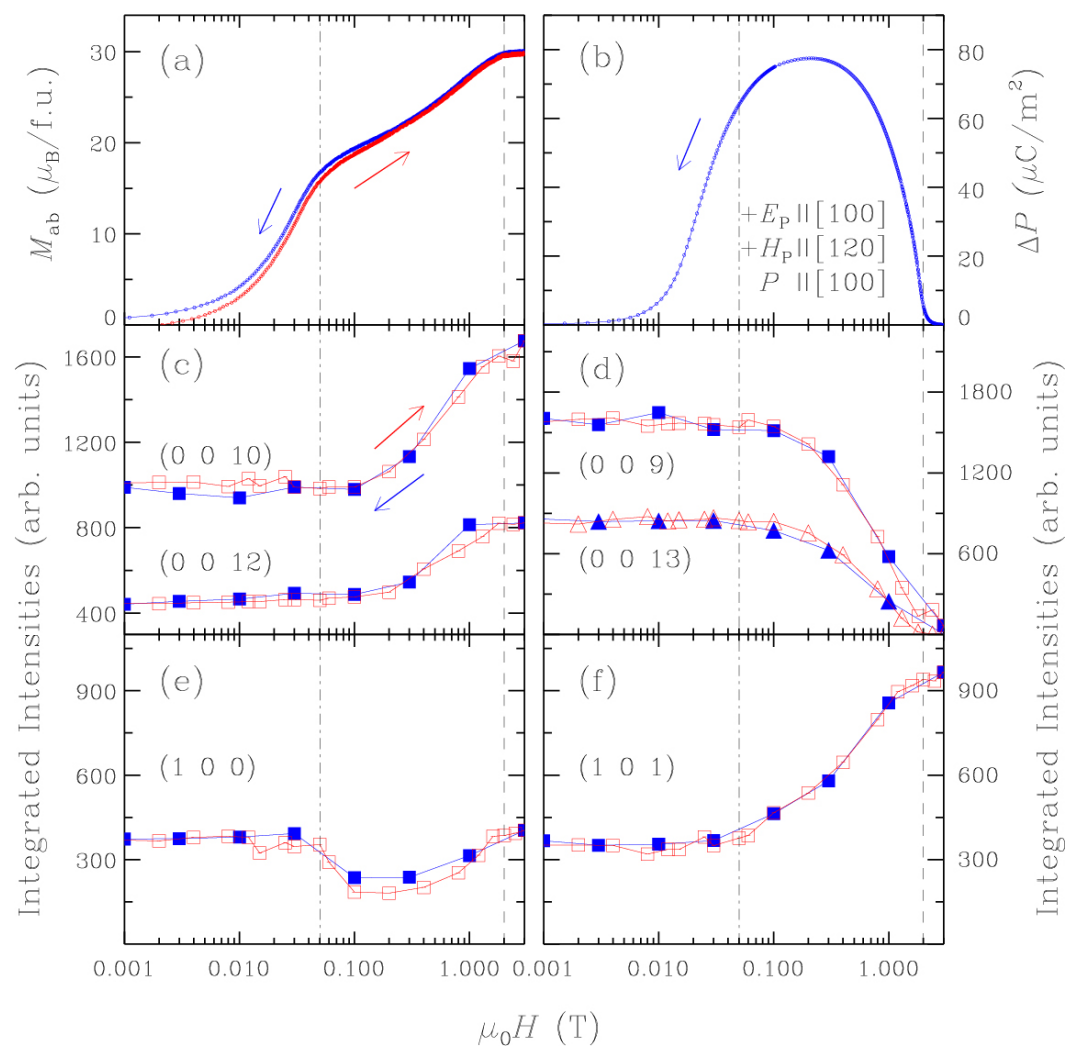

Figure 3: Field-dependent changes at $10 \mathrm{~K}$ in (a) dc magnetization, (b) electric polarization, and (c-f) integrated Bragg peak intensities. The arrows indicate the directions of the magnetic field changes during successive measurements. In (c) - (f), the empty and solid symbols represent data measured during ramping up and down, respectively. The dash-dotted and dashed vertical lines mark the field values discussed in the text. 